BNL -97930

DE92 040681

\title{
Localized Control of the Orbit in the RHIC Insertions
}

\author{
S. Ohnuma
}

August 1992

\section{DISCLAIMER}

This report was prepared as an acoount of work sponsored by an agency of the United States Government. Neither the United States Goveinment nor any agency thereof, nor any of their employees, makes any warranty, express or imy/ied, or assumes any legal liability or responsibility for the accuracy, completeness, or usefulness of any information, apparatus, product, or process disclosed, or represents that its use would not infringe privately owned rights. Reference herein 10 any specific commercial product, process, or service by trade name, trademark, manufacturer, ot otherwise does not necessarily constitute or imply its endorsement, rexommendation, or favoring by the United States Government or any agency thereof. The yiews and opinions of authors expressed herein do not necessarily state or reflect those of the United States Government or any agency thereof.

\section{RH I C P R O J E C T}

\author{
Brookhaven National Laboratory \\ Associated Universities, Inc. \\ Upton, NY 11973
}

Under Contract No. DE-AC02-76CH00016 with the UNITED STATES DEPARTMENT OF ENERGY 


\title{
Localized Control of the Orbit in the RHIC Insertions
}

\author{
S. Ohnuma* \\ Accelerator Development Department \\ Brookhaven National Laboratory \\ August 10, 1992
}

\section{Summary}

It is proposed here that, for RHIC92 insertions, we remove the corrector from $Q 1$ and the beam position monitor (BPM) from $Q 2$ in order to alleviate difficulties associated with the physical layout of the quadrupole triplet (Q1-122-Q3). Furthermore, it is suggested that there should be both (horizontal and vertical) types of BPMs at each end of the free space between Q3 and Q4 and between Q7 ard Q8 so that one can measure the direction of the closed orbit. With this model, a localized control of the bearn position and angle at the interaction point (IP) with either four or six correctors has been investigated. Similarly, a control of the orbit within an insertion for minimizing the orbit displacements at seven (or eight) BPM locations with nine (or ten) correctors in each transverse direction has been studied. Examples are given for the beta at $I P=2 \mathrm{~m}, 10 \mathrm{~m}, 20 \mathrm{~m}$, and $200 \mathrm{~m}$. It is shown. that the design value of the integrated field strength of $0.3 \mathrm{~T}$-m for each corrector should be sufficient for the tasks considered here except for some cases with extreme parameter values. At the same time, it is emphasized that the overall correction of the closed orbit for the entire ring (arcs and insertions) should be re-examined for RHIC92 lattice with the proposed arrangement of correctors and BPMs.

\section{Introduction}

In an at tempt to make the quadrupole triplet (Q1-Q2-Q3) in insertions as compact as possible, the design of RHIC92 ${ }^{1}$ may have generated a certain difficulty in the physical layout of the triplet and its neighboring dipole D0. ${ }^{2}$ This is shown in Fig. 1 as RHIC92 (upper figure) where the distance betweer two adjacent quadrupoles is less than $1^{\prime \prime}$. The phy'sical distance between $Q 1$ and dipole Do is not yet precisely specified but the present design is unlikely to provide an adequate space there either.

In order to ease the difficulty, it is proposed here that we remove the corrector from Q1 and the BPM from Q2, and switch the position of BPM and corrector attached to Q3

\footnotetext{
* Permanent address: University of Houston, Houston, TX 77204-550e
} 
so that the BPM now faces the BPM attached to Q4. There will be a space of $36.7 \mathrm{~m}$ long, which is free of magnets. Furthermore, it is proposed that there should be both horizontal and vertical BPMs there as well as at Q7 and Q8, again facing each other at a distance of $11.5 \mathrm{~m}$. The overall layout is shown in Fig. 2 where BPMs on both sides of IP are the ones attached to common dipoles DX. Since the resolution of BPM is expected to be better than $0.1 \mathrm{~mm}$, a change as small as $0.01 \mathrm{mr}$ in the orbit direction can be detected without much difficulty.

With this proposed arrangement of correctors and BPMs, two tasks have been examined to find out how much integrated field strength is needed for each corrector. Cases studied here are with $\beta^{*}=2 \mathrm{~m}, 10 \mathrm{~m}, 20 \mathrm{~m}$, and $200 \mathrm{~m}$. It is expected that $\beta^{*}$ will be $10 \mathrm{~m}$ at all insertions initially with the possible exception of one being $\beta^{*}=20 \mathrm{~m}$. When data taking is ready, $\beta^{*}$ will be squeezed down to $2 \mathrm{~m}$ from $10 \mathrm{~m}$ at one or two IPs, and increased from $20 \mathrm{~m}$ to $200 \mathrm{~m}$ or so for the small-angle experiment. There is of course a possibility of $\beta^{*}=1 \mathrm{~m}$ but this has not been considered in this study.

Task A. Locally control $\left(x, x^{\prime} ; y, y^{\prime}\right)$ at IP.

This is to maintain the proper collision but also to separate the two beams when such a separation is beneficial.

Task B. Minimize the orbit displacements at BPMs within an insertion locally.

This is different from a global correction of the closed orbit in the entire ring. One might question the necessities of such a localized orbit correction when global corrections are believed to be possible with a few iterations. In a way, this is simply a matter of convenience. When $\beta^{*}$ is changed from $10 \mathrm{~m}$ to $2 \mathrm{~m}$ or from $20 \mathrm{~m}$ to $200 \mathrm{~m}$, the largest change in the closed orbit will be within that particular insertion. It will be certainly convenient to confine the needed adjustment within the insertion instead of repeating a global correction everywhere. The amount of correction for this should be substantially less than the initial correction for the first turn.

\section{Existing Reports on the RHIC Closed Orbit}

\#1. J. Milutinovic and A.G. Ruggiero, "Closed Orbit Analysis for RHIC", AD/RHICAP. 78 (February 21, 1989); AD/RHIC-51 (March 20, 1989).

The lattice used for this study is RHIC88I with $\beta^{*}=3 \mathrm{~m}$ at all IPs. Even with the expected $\mathrm{rms}$ displacement as large as $50 \mathrm{~mm}$ in insertions, they found that the required maximum corrector strength is $0.17 \mathrm{~T}-\mathrm{m}$ at $(\mathrm{B} \rho)=850 \mathrm{I}-\mathrm{m}$. This is less than $60 \%$ of the design value. They emphasize the importance of establishing a good closed orbit with all sextupoles turned off in order to avoid complications. It is not clear from these 
reports how correctors and BPMs are arranged in insertions. The finding that only 0.17 T-m is needed to correct the orbit everywhere even at the highest energy is somewhat surprising. This should be re-examined for RHIC92 with the proposed arrangement of correctors and BPMs. To be re-examined also are the expected magnitude of various errors contributing to the orbit displacement, namely, the variation in the integrated field strength of main dipoles, the rotation of dipoles, and the transverse displacements of quadrupoles. Especially important is a reliable estimate of errors associated with quadrupole triplets in insertions.

\#2. A.G. Ruggiero, "Analysis and Correction of the Closed Orbit Distortion for RHIC", AD/RHIC/AP-90 (April 1990).

This is a review article containing discussions on many interesting subjects related to the closed orbit correction. There is again a statement, "The method once applied indeed provides zero closed orbit distortion at any observed location with a maximum required strength of the correctors about half of what is available." One important question raised in this article is the definite possibility of one (or more) BPMs not working. This would be particularly serious if the non-working BPM is in an insertion.

There is an observation (the last paragraph, p. 13) regarding a possible tune shift arising from the sextupole where the orbit remains uncorrected because of the non-working BPM at that location in an arc. It is possible that this can be used to find the displacement if tunes can be measured with the accuracy of 0.0005 with many sextupoles off. For example, at $\beta=50 \mathrm{~m}$, the expected change in the ture is 0.0005 horizontally and 0.0009 vertically when the orbit displacement is $1 \mathrm{~mm}$. The sextupole strength is assumed to be $\left(B^{\prime \prime} l / B \rho\right)=0.13 / \mathrm{m}^{2}$ (horizontal stations) and $-0.23 / \mathrm{m}^{2}$ (vertical stations) which make the natural chromaticity zero in both directions when $\beta^{*}=10 \mathrm{~m}$ in all insertions. It should be possible to change the orbit displacement near the non-working BPM with the neighboring sextupole on and off and measure the resulting change in the tune. The assumption here is that the tune change arising from a.l other sextupoles, which are in series with the one under consideration, is negligible. A computer simulation of this possibility should be explored. It will be a challenge to develop a practical strategy along this line when two (or even more) BPMs are not working in arcs.

\#3. J. Milutinovic and A.G. Ruggiero, "First Turn Around Strategy for RHIC", BNL45516 (May 1991); AD/RHIC-91.

This should definitely be repeated for RHIC92 with the proposed arrangement of corlectors and BPMs in insertions. Because of its somewhat irregular layout, the strategy employed in this report might require some modifications for RHIC92. It might be prudent 
that the work be done by two people (or two groups of people) independently in order to avoid any errors (which are all too common in this type of study).

\#4. S.Y. Lee and S. Tepikian, "Closed Orbit Analysis for RHIC Insertion", AD/RHIC. 64 (February 1990); "Analytic Closed Orbit Analysis for RHIC Insertion", BNL45523, AD/RHIC-98 (May 1991).

These studies are for RHIC91 and their main concern is the dynamic effect arising from power supply ripples on the orbit displacement at IP as well as at a few insertion quadrupoles. As such, their essential contribution is the tables of " $m_{12}$ " and " $m_{34}$ ", the relevant elements of the $4 \times 4$ transfer matrix between two points in the insertion for various values of $\beta^{*}$. Such tables are of course an essential ingredient of applications programs to be used in the control room. An important question related to this is the possibility of a feedback system, fast and slow, which enables a continuous monitoring and correction of the orbit at IPs.

In preparing a table of " $\mathrm{m}_{12}$ " and " $\mathrm{m}_{34}$ ", there is always a question of non-vanishing " $\mathrm{m}_{14}$ " and " $\mathrm{m}_{32}$ ", that is, the effect of linear coupling between two points under consideration. This is not addressed in the study of Lee and Tepikian. One uncertainty at present is the expected rotation of the field axis in quadrupoles within a triplet where the product $\beta_{x} \beta_{y}$ can be very large. For example, with $\beta^{*}=2 \mathrm{~m}, \beta^{\prime}$ s are $660 \mathrm{~m}$ and $275 \mathrm{~m}$ at Q2. If the roll angle is $1 \mathrm{mr}$ and the horizontal displacement is $10 \mathrm{~mm}$ at $\mathrm{Q} 2$, the resulting vertical closed orbit displacement will be $2 \mathrm{~mm}$ at the same quadrupole and up to $0.6 \mathrm{~mm}$ in arcs. It should still be possible to control the orbit within the insertion by alterniately applying the horizontal and vertical correction procedures. A related question is whether one can locally detect a gross rotational misalignment of quadrupoles in an insertion from the orbit measurement (including its direction at Q3-Q4 or at Q7-Q8) alone. This is yet another subject for a simulation study.

\section{Expected Impact of the Modification}

The slight $(\simeq 30 \mathrm{~cm})$ shift of quadrupole triplets away from IP should not affect the beam dynamics in any appreciable degree. The main concern here is the impact of missing BPM at Q2 and missing corrector at Q1.

a) Missing BPM at, Q2.

Since the phase advance between $Q 1$ and Q3 is so small, usually less than a few degrees, $B P M$ at $Q 2$ cannot provide information not available from the BPMs at $Q 1$ and Q3. The relative sensitivity of a BPM is proportional to the sq. me root of $\beta$ at that location and this gives a certain advantage to the $B P M$ at $Q 2$ over others: 


$\begin{array}{lcc} & \sqrt{\beta(\mathrm{Q} 1) / \beta(\mathrm{Q} 2)} & \sqrt{\beta(\mathrm{Q} 3) / \beta} \overline{\mathrm{Q} 2)} \\ \beta^{*}=2 \mathrm{~m} & 0.73 & 0.65 \\ \beta^{*}=10 \mathrm{~m} & 0.74 & 0.64 \\ \beta^{*}=20 \mathrm{~m} & 0.80 & 0.62 \\ \beta^{*}=200 \mathrm{~m} & 0.88 & 0.63\end{array}$

Nevertheless, this advantage is not overwhelming and healthy BPMs at Q1 and Q3 should be able to take care of any tasks.

b) Missing corrector at Q1.

Again the phase difference within a triplet is so small that the only impact of this is the need to do a work with one corrector at Q3 instead of two correctors at Q1 and Q3. (The corrector at Q2 is in the "wrong" direction.) The relevant quantity is therefore the ratio

$$
\begin{aligned}
& R=\frac{\text { " } \mathrm{m}_{12} \text { " or " } \mathrm{m}_{34} \text { " from } \mathrm{Q} 3 \text { to a BPM }}{\mathrm{m}_{12} \text { " or " } \mathrm{m}_{34} \text { " from } \mathrm{Q1} \text { and } \mathrm{Q3} \text { combined to the same BPM }} \\
& \beta^{*}=2 \mathrm{~m} \quad 10 \mathrm{~m} \quad 20 \mathrm{~m} \quad 200 \mathrm{~m} \\
& R=0.53-0.59 \quad 0.54-0.63 \quad 0.57-0.65 \quad 0.48-0.58
\end{aligned}
$$

It is certainly true that, by removing the corrector at $\mathrm{Q} 2$, we need more field strength at the Q3 corrector. It should be pointed out, however, that adding a corrector at Q2 does not really help a deficiency at other correctors. As long as the Q3 corrector can do all the tasks, there is no need to have one more (equivalent) corrector at Q1. Since the results reported here are limited to localized controls of the orbit, this point must be examined for the first-turn strategy and for the global orbit correction with RHIC92 before making the final decision on the maximum integrated field of insertion correctors.

\section{Localized Controls of the Orbit}

In what follows, the kick angle of a corrector is always given in $\mathrm{mr}$. The conversion from the angle $\theta$ in $\mathrm{mr}$ to the corrector field $\mathrm{B}$ in Tesla is

$$
B=(0.20-1.7) \theta \quad \text { for }(B \rho)=(100-840) \mathrm{T}-\mathrm{m}
$$

with the corrector magnetic length $=0.50 \mathrm{~m}$. For correctors attached to Q1, Q2 or Q3 with $13 \mathrm{~cm}$ aperture (instead of $8 \mathrm{~cm}$ ), the effective magnetic length may be $\sim 10 \%$ less and the corrector field for the same kick angle may have to be increased by that amount.

Since nonlinear effects are not included in finding the kick angle, its value always scales linearly with the corresponding orbit change. 


\subsection{Task A: Localized control of $\left(x, x^{\prime} ; y, y^{\prime}\right)$ at IF}

It is desirable to have a continuous monitoring and adjustment of the orbit at IP during collision. For this, one would primarily depend on the BPMs on both sides of IP, attached to DXI and DXO. From the change in the sum and difference of two BPM readings, one cans get the change in $x$ (or $y$ ) and in $x^{\prime}$ (or $y^{\prime}$ ) at IP, respectively. Since the distance between two BPMs is $19 \mathrm{~m}$, a change of $2 \mathrm{~mm}$ corresponds to a change of $0.1 \mathrm{mr}$, which is less than the rms value of he beam angular spread $(0.18 \mathrm{mr})$ with $\beta^{*}=2 \mathrm{~m}$ and emittance $(95 \%$, unnormalized $)=0.4 \pi \mathrm{mm}-\mathrm{mr}$.

Another reason for the local control of orbit at IP is to introduce an intentional separation of two beams at an IP when surh a separation is beneficial. The amount of separation may be of the order of $(5-10) \sigma, \sigma$ being the rms beam size.

Examples: $\quad \epsilon_{N}(95 \%)=10 \pi \mathrm{mm}-\mathrm{mr}$, momentum factor $(\gamma \beta)=10$

$$
\begin{aligned}
\sigma & =1.3 \mathrm{~mm} \text { for } \beta^{*}=10 \mathrm{~m} \\
& =1.8 \mathrm{~mm} \text { for } \beta^{*}=20 \mathrm{~m} \\
\epsilon_{N}(95 \%)= & 40 \pi \mathrm{mm}-\mathrm{mr}, \text { momentum factor }(\gamma \beta)=100 \\
\sigma & =0.82 \mathrm{~mm} \text { for } \beta^{*}=10 \mathrm{~m} \\
& =1.15 \mathrm{~mm} \text { for } \beta^{*}=20 \mathrm{~m}
\end{aligned}
$$

Correctors to be used are at $(\mathrm{Q} 7, \mathrm{Q} 5, \mathrm{Q3})$ on one side of IP and at $(\mathrm{Q} 2, \mathrm{Q} 4, \mathrm{Q} 6)$ on the other side. In order to control $x$ (or $y$ ) and $x^{\prime}$ (or $y^{\prime}$ ) independently at IP and to co-fine the change within a certain area, we need at least four in dependent correctors.

a) If four correctors are used, the best combination is (Q5,Q3;Q2,Q6).

$\begin{array}{llll}\text { max. kick angle } & \Delta= \pm 5 \mathrm{~mm} & \Delta^{\prime}= \pm 0.5 \mathrm{mr} & \Delta= \pm 5 \mathrm{~mm} \text { and } \\ \beta^{*}=2 \mathrm{~m} & 0.147 & 0.181 & \Delta^{\prime}= \pm 0.25 \mathrm{mr} \\ \beta^{*}=10 \mathrm{~m} & 0.138 & 0.216 & 0.175 \\ \beta^{*}=20 \mathrm{~m} & 0.123 & 0.260 & 0.187 \\ \beta^{*}=200 \mathrm{~m} & 0.087 & 0.376 & 0.223\end{array}$

The combination (Q5,Q3;Q2,Q4) is also possible if a change in $x^{\prime}$ (or $y^{\prime}$ ) is not required; the maximum kick angle for $\Delta=5 \mathrm{~mm}$ is less than $0.2 \mathrm{mr}$. When this is combined with a change of $\Delta^{\prime}=0.25 \mathrm{mr}$, the requirement for $\mathrm{Q} 4$ kicker goes beyond the maximurn integrated field of $0.3 \mathrm{~T}-\mathrm{m}$.

b) If all six correctors are used the maximum kick angle is reduced but not by a significant amount. 


$\begin{array}{llll} & \Delta= \pm 5 \mathrm{~mm} & \Delta^{\prime}= \pm 0.5 \mathrm{mr} & \begin{array}{l}\Delta= \pm 5 \mathrm{~mm} \text { and } \\ \Delta^{\prime}= \pm 0.25 \mathrm{mr}\end{array} \\ \beta^{*}=2 \mathrm{~m} & 0.122 & 0.100 & 0.130 \\ \beta^{*}=10 \mathrm{~m} & 0.120 & 0.137 & 0.145 \\ \beta^{*}=20 \mathrm{~m} & 0.116 & 0.176 & 0.140 \\ \beta^{*}=200 \mathrm{~m} & 0.060 & 0.320 & 0.175\end{array}$

When a change in $x^{\prime}$ or $y^{\prime}$ is introduced, one must of course watch out for large orbit displacements within the affected area. For example, with $\Delta x^{\prime}$ (or $\Delta y^{\prime}$ ) $= \pm 0.25 \mathrm{mr}$ but $\Delta x$ (or $\Delta y)=0$, the displacement within triplets can be as large as $\sim 10 \mathrm{~mm}$ and this may significantly affect the dynamic aperture of the ring.

Regardless of what combination of correctors is used, the position readings of BPMs at Q7 and Q8 must be checked to confirm the localization of orbit disturbance.

\subsection{Task B: Minimizing the orbit displacements within an insertion}

This can be done globally together with arcs and other insertions as Milutinovic and Ruggiero have done. A localized orbit correction is convenient when $\beta^{*}$ is changed and the resulting orbit change is large within that particular insertion.

Nine correctors are used to control the orbit displacements at seven BPMs within an insertion. If one more corrector is used, the orbit displacement at one more BPM location can be controlled and this is indicated in parenthesis in the following list:

6 o'clock horizontal or 8 o'clock vertical

corrector at Q11, 9, 7, 5, 3; Q2, 4, 6, 8, (10)

$\mathrm{BPM}$ at $\mathrm{Q} 9,7,5,3 ; \mathrm{Q} 1,4,6,(8)$

6 o'clock vertical or 8 o'clock horizontal

corrector at Q10, 8, 6, 4, 2; Q3, 5, 7, 9, (11)

$\mathrm{BPM}$ at $\mathrm{Q} 8,6,4,1 ; \mathrm{Q} 3,5,7,(9)$

There is a fundamental question, "What should be the largest displacement to be handled by this scheme?". In order to answer this question properly, one must have a realistic estimate of the errors within an insertion. It is also necessary to find the ability of a global correction in RHIC92. The necessity for repeating the work by Milutinovic and Ruggiero has already been emphasized. Since all quantities scale linearly, it does not matter here what we take as the displacement. For examples given below, it is assumed that the orbit displacements to be corrected locally within each insertion are

$$
\mid \text { displacement } \mid \leq(5 \mathrm{~mm}) \quad \sqrt{\beta / 50 \mathrm{~m}} ; \text { uniform distribution. }
$$


Uniform distribution is used here instead of Gaussian distribution since the source of errors under consideration is limited to a relatively small number of magnets. Altogether 100 random samples have been used for each $\beta^{*}$ with the following results for the maximum kick angle of any of nine correctors. Approximately $65 \%$ of 100 cases require maximum kick angles within the rms angles, which are listed together with the largest in any of 100 cases.

$\begin{array}{lllll}\beta^{*}= & 2 \mathrm{~m} & 10 \mathrm{~m} & 20 \mathrm{~m} & 200 \mathrm{~m} \\ \max . & 0.75 & 0.51 & 0.46 & 0.71 \\ \mathrm{rms} & 0.30 & 0.21 & 0.20 & 0.30\end{array}$

The maximum kick angle is always at Q4 corrector. If Q4 corrector is not included in the tabulation, the maximurn kick angles are:

$\begin{array}{lllll}\beta^{*}= & 2 \mathrm{~m} & 10 \mathrm{~m} & 20 \mathrm{~m} & 200 \mathrm{~m} \\ \max . & 0.50 & 0.28 & 0.36 & 0.54 \\ \text { rms } & 0.22 & 0.13 & 0.16 & 0.25\end{array}$

One is tempted to say that the maximum design value of $0.3 \mathrm{~T}$-m for the corrector integrated field is sufficient to take care of the task under consideration unless the situation is extreme. The final judgement must, however, depend on a more reliable estimate of the magnitude of various errors in inserticn magnets than the one "talked about" at present.

An alternative but (almost) equivalent way to control the displacement locally within an insertion is the familiar 3-bump method. A small but finite distance between corrector: and BPM attached to the same quadrupole makes it necessary to modify the 3-bump formulas slightly but this does not change the overall picture. It has been found out that, of all possible combinations of three neighboring correctors, the maximum kick angle per unit displacement is again at Q4 in (Q2-Q4-Q6) bump:

max. kick angle at Q4 for $5 \mathrm{~mm}$ displacement at Q4 BPM

$$
\begin{array}{lllll}
\beta^{*}= & 2 \mathrm{~m} & 10 \mathrm{~m} & 20 \mathrm{~m} & 200 \mathrm{~m} \\
0.403 & 0.247 & 0.197 & 0.205
\end{array}
$$

The large angle with $\beta^{*}=2 \mathrm{~m}$ is understandable since $\beta$ at $\mathrm{Q} 4$ is abnormally small in spite of the fact that it is in the focusing direction: $\beta=17.4 \mathrm{~m}$ at BPM, $20.6 \mathrm{~m}$ at corrector. Presumably, the displacement is likely to be small at $Q 4$ and the requirement for the corrector kick angle may not be as large as at other locations. 


\section{Concluding Remarks}

In the design of almost any accelerators, most decisions are likely to be a matter of judgment. To be sure, the judgment must always be based on a reliable quantitative evaluation of all the relevant quantities. Even then, one is often forced to compare "apples with oranges", and this makes it inevitable that one's taste and prejudice often play an important role in making the final decision. Because of this, the role of an outsider should be strictly limited to supplying factual data as much as he can within a given (often short) period.

Factual data which are vital but missing in this report, and which should be acquired soon for the final decision, are:

1. Evaluation of the expected magnitude of all types of errors that are relevant to the closed orbit displacement, in particular those associated with the insertion magnets.

2. Capability of a global orbit correction and the first-turn strategy for RHIC92 with the proposed layout of correctors and BPMs in insertions.

\section{References}

1. S. Tepikian and M. Harrison, "The Conceptual Design of the RHIC 92 Insertions", AD/RHIC/AP-103 (June 1992).

2. Charles Rufer, private communication. 


\section{Appendix . Orbit Displacements Generated by $\beta^{*}$ Squeeze}

In the discussion of Task B: Minimizing the orbit displacements within an insertion, it is assumed without any justification that the displacements to be corrected locally within each insertion are

$$
\mid \text { displacement } \mid \leq(5 \mathrm{~mm}) \times \sqrt{\beta / 50 \mathrm{~m}} ; \text { uniform distribution }
$$

The factor, $5 \mathrm{~mm}$, was used as a convenient scale factor and not as something we would have from the expected errors. After all, the results presented are linear and the scaling is a trivial matter. Nevertheless, there were some unhappiness expressed by several people when I gave a talk on this subject.

When $\beta^{*}$ is squeezed from $10 \mathrm{~m}$ to $2 \mathrm{~m}$ at one insertion, for example, effects on the closed orbit will be everywhere around the ring, arcs as well as all the insertions. Since the resulting disturbance to the closed orbit is proportional to the square-root of the product of two $\beta \mathrm{s}$, one at the source of misalignment and the other at the observation point, the maximum effect will be in that insertion where $\beta^{*}$ is squeezed. Moreover, the change in $\beta$ is predominantly at quadrupole triplets (and at dipoles DX and DO). With this in mind, I have checked the following situation.

Assume $\beta^{*}$ is either $10 \mathrm{~m}$ or $20 \mathrm{~m}$ at all insertions and the closed orbit displacements are corrected to some acceptable level by means of either a global or a local correction. Now squeeze $\beta^{*}$ from $10 \mathrm{~m}$ to $2 \mathrm{~m}$ at one insertion and evaluate the change in the closed orbit displacements at quadrupole triplets in that same insertion. In doing this, take into account the quadrupole misalignments within triplets only. There are two triplets, aach with three quadrupoles. Assume that the misalignment (magnetic and geometrical) of each quadrupole is composed of two parts:

$$
\text { misalignment }=(\text { cormmon })+(\text { relatjve })
$$

where (common) is the error in installation of the triplet assembly (geometrical only?) and (relative) is the uncorrelated part of each quadrupole misalignment (magnetic and geometrical). Obviously, if the total misalignment is predominantly (common), there will be a substantial amount of cancellation since the phase advance within eirch triplet is very small and the summation of $\left(B^{\prime} l\right)$ for three quadrupoles is much less than the individual value of $\left(B^{\prime} l\right)$.

What we are interested in is the change in displacements when $\beta^{*}$ is squeezed. In order to see if my choice of $5 \mathrm{~mm}$ (see above) is reasonable, the factor

$$
\text { |displacement } \mid / \sqrt{(\beta / 50 \mathrm{~m})} \quad \text { in } \mathrm{mm}
$$


is tabulated for various combinations of (common) and (relative) misalignments. The value of $\beta$ used in this expression is the one corresponding to $\beta^{*}=2 \mathrm{~m}$. In each case, 100 random samples have been taken and the rms values of the change in displacement at each quadrupole are tabulated. Approximately 70 cases out of 100 are within the rms values.

A. (common) is uniformly distributed within $\pm \Delta$; (relative) is Gaussian distribution with the cutoff of $2.5 \sigma$.

\begin{tabular}{|c|ccc|}
\hline & $\Delta=0 \mathrm{~mm}$ & $0.15 \mathrm{~mm}$ & $0.25 \mathrm{~mm}$ \\
\hline$\sigma=0 \mathrm{~mm}$ & 0 & $0.77 \sim 0.78$ & $1.28 \sim 1.30$ \\
& & $(1.01 \sim 1.23)$ & $(1.69 \sim 2.06)$ \\
$0.10 \mathrm{~mm}$ & $3.00 \sim 3.06$ & $3.01 \sim 3.08$ & $3.13 \sim 3.20$ \\
& $(3.86 \sim 4.58)$ & $(3.95 \sim 4.65)$ & $(4.15 \sim 4.88)$ \\
& & & \\
$0.15 \mathrm{~mm}$ & $4.50 \sim 4.58$ & $4.48 \sim 4.57$ & $4.54 \sim 4.62$ \\
& $(5.80 \sim 6.87)$ & $(5.84 \sim 6.89)$ & $(5.97 \sim 7.02)$ \\
& & & \\
$0.25 \mathrm{~mm}$ & $7.49 \sim 7.60$ & $7.45 \sim 7.60$ & $7.47 \sim 7.62$ \\
& $(9.66 \sim 11.5)$ & $(9.67 \sim 11.4)$ & $(9.74 \sim 11.5)$ \\
\hline
\end{tabular}

Numbers in parenthesis are with two IPs (6 o'clock and 8 o'clock) simultaneously squeezed from $10 \mathrm{~m}$ to $2 \mathrm{~m}$.

B. Both (common) and (relative) are Gaussian with the cutoff of $2.5 \sigma$.

$$
\begin{array}{ll}
\sigma(\text { common })=\sigma(\text { relative })=0.25 / \sqrt{2}: & (4.85 \sim 4.91) \mathrm{mm} \\
\sigma(\text { common })=0.25 \times \sqrt{2 / 3}, \sigma(\text { relative })=0.25 \times \sqrt{1 / 3}: & (4.15 \sim 4.20) \mathrm{mm}
\end{array}
$$

If two neighboring IPs are squeezed simultaneously, the factors are $(7.01 \sim 8.51) \mathrm{mm}$ and $(5.90 \sim 7.15) \mathrm{mm}$.

It is not surprising that the displacement is determined almost totally by the relative misalignment and the common misalignment is important only when the relative misalignment is much smaller than the common misalignment. Results given here show that the choice of $5 \mathrm{~mm}$ is (accidentally) not at all unreasonable when $\beta^{*}$ is squeezed from $10 \mathrm{~m}$ to $2 \mathrm{~m}$ at one IP only. With two IPs, $5 \mathrm{~mm}$ may be an underestimate, but this again depends veiy much on the amount of relative misalignment. 


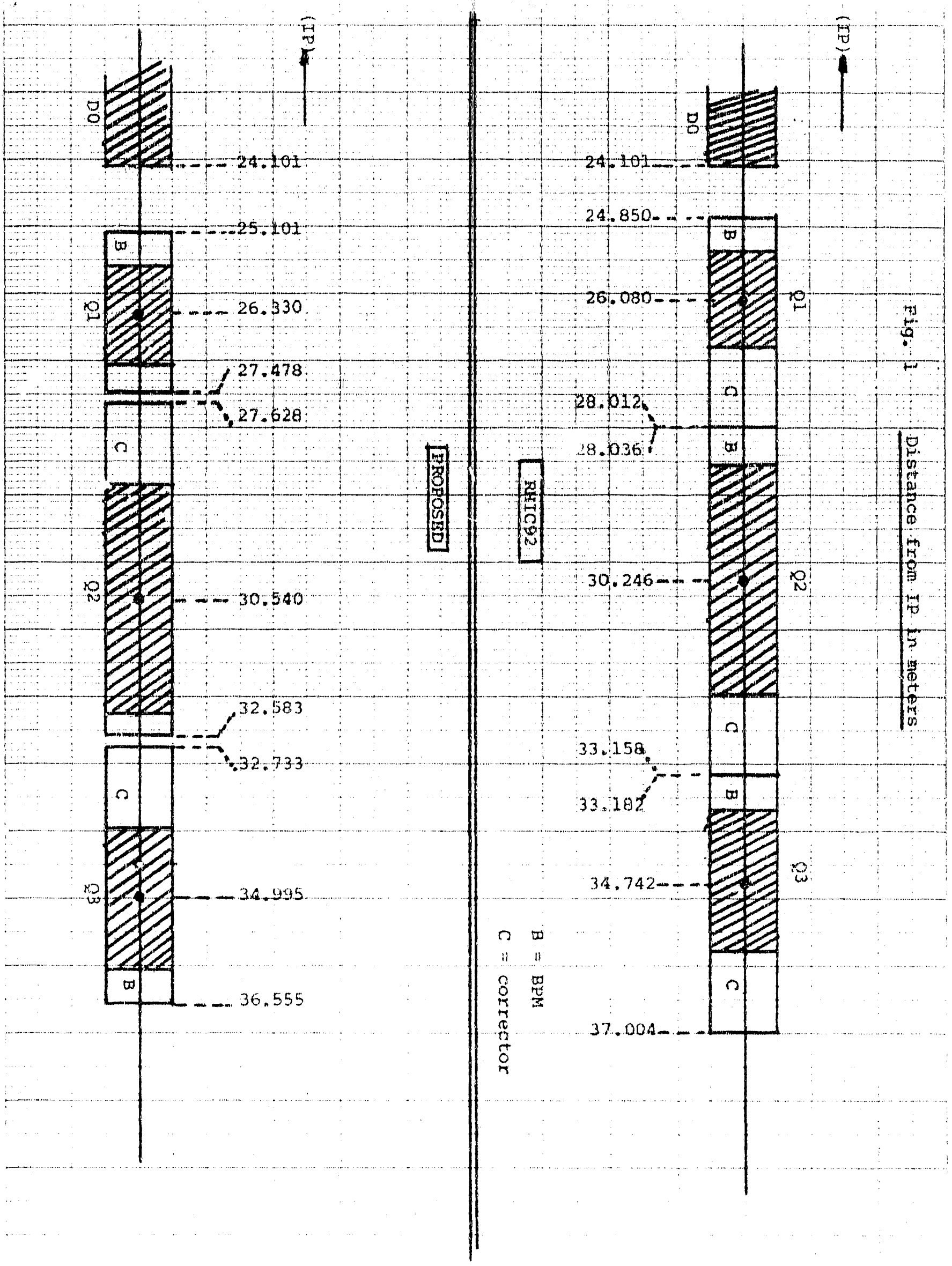



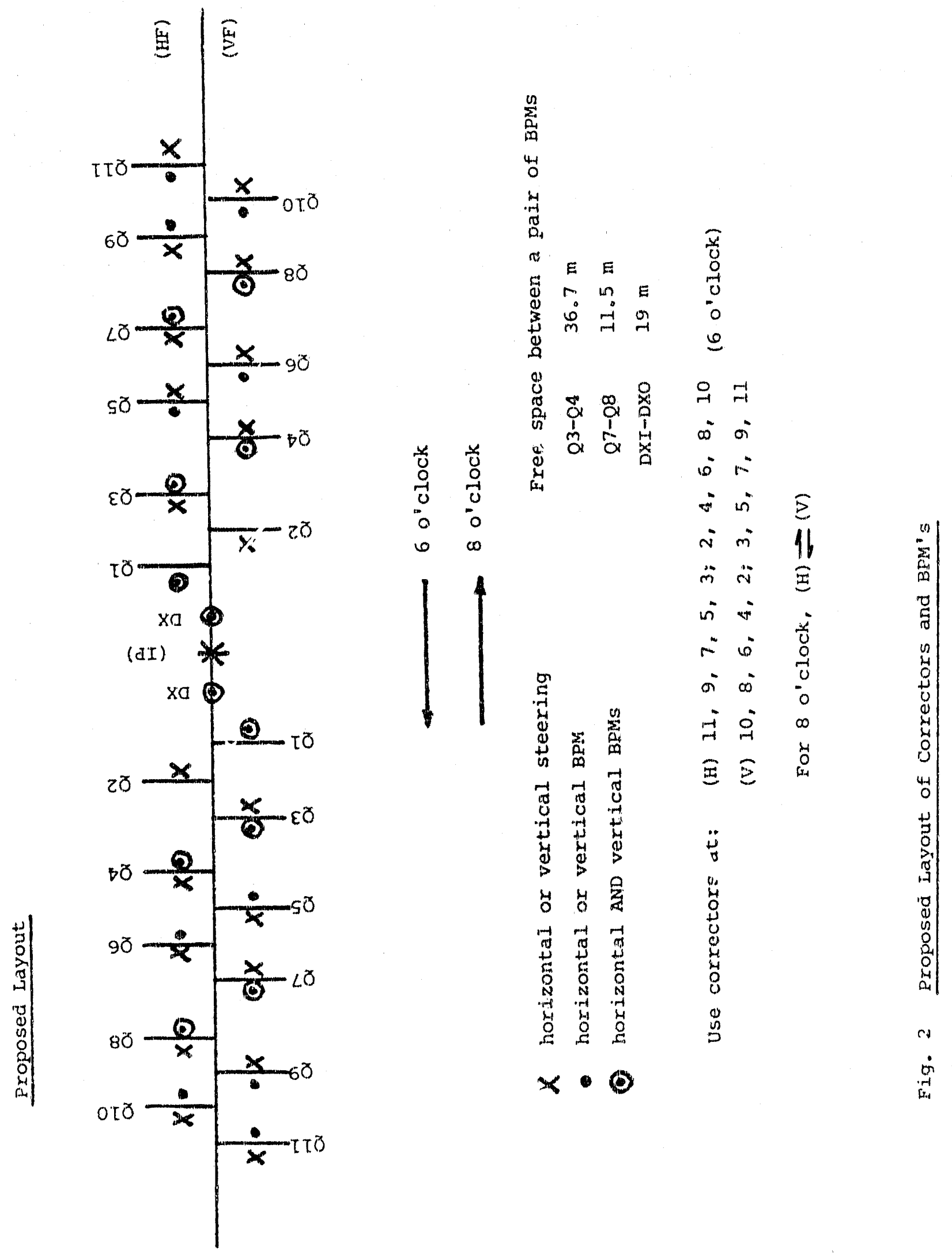

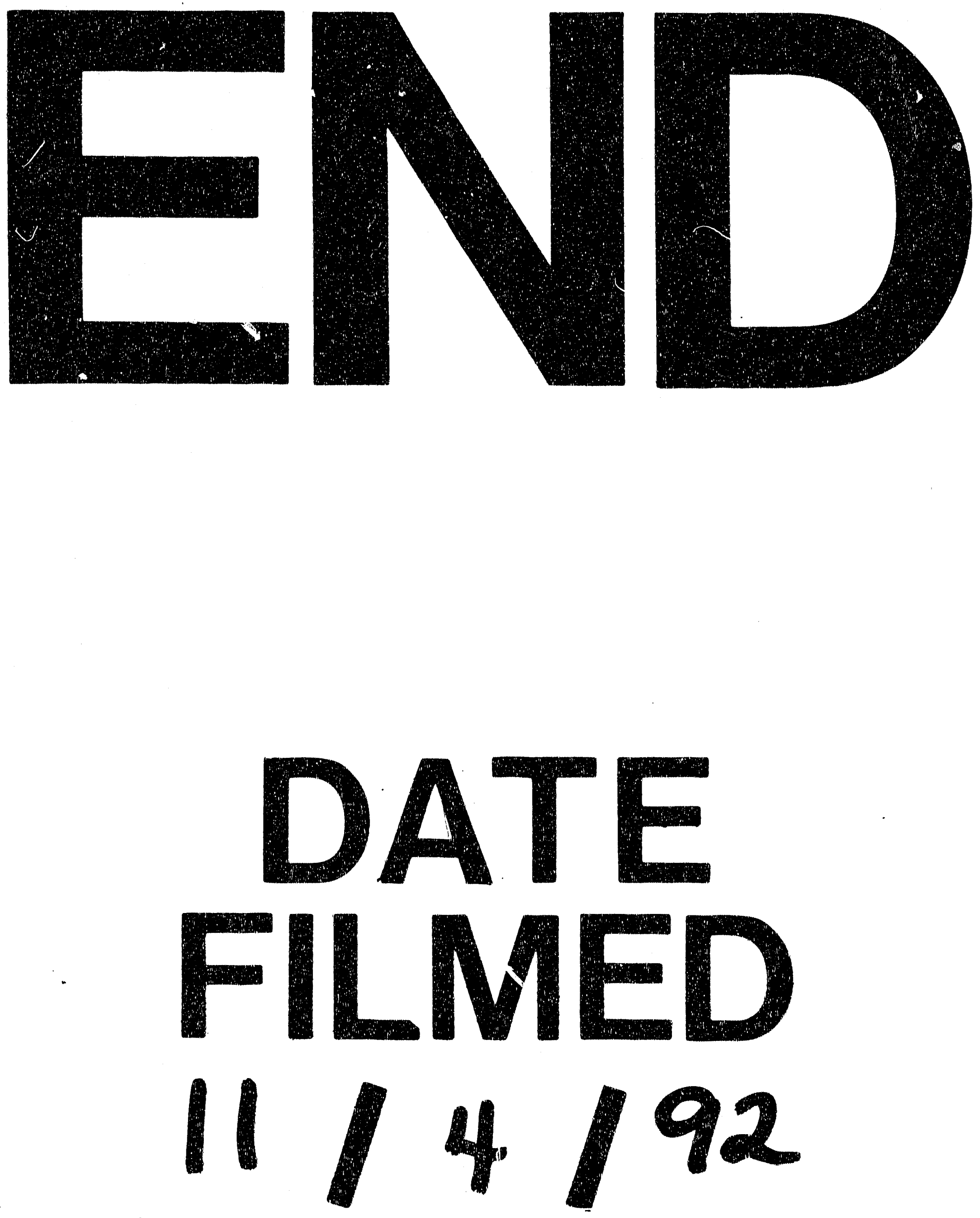
1

1 